\title{
Structural and Functional Brain Patterns of Non-Motor Syndromes in Parkinson's Disease
}

\author{
Tino Prell* \\ Department of Neurology, Jena University Hospital, Jena, Germany
}

Parkinson's disease (PD) is a common, progressive and multisystem neurodegenerative disorder characterized by motor and non-motor symptoms. Advanced magnetic resonance imaging, positron emission tomography, and functional magnetic resonance imaging can render the view toward understanding the neural basis of these non-motor syndromes, as they help to understand the underlying pathophysiological abnormalities. This review provides an up-to-date description of structural and functional brain alterations in patients with PD with cognitive deficits, visual hallucinations, fatigue, impulsive behavior disorders, sleep disorders, and pain.

OPEN ACCESS

Edited by:

Jan Kassubek,

University of Ulm, Germany

Reviewed by:

Alessandro Tessitore,

Università degli Studi della

Campania "Luigi Vanvitelli"

Caserta, Italy

Martin Gorges,

University of UIm, Germany

*Correspondence:

Tino Prell

tino.prel/@med.uni-jena.de

Specialty section:

This article was submitted to

Applied Neuroimaging,

a section of the journal

Frontiers in Neurology

Received: 14 December 2017 Accepted: 26 February 2018

Published: 12 March 2018

Citation:

Prell T (2018) Structural and

Functional Brain Patterns of

Non-Motor Syndromes in

Parkinson's Disease.

Front. Neurol. 9:138.

doi: 10.3389/fneur.2018.00138
Keywords: Parkinson's disease, cognition, imaging, positron emission tomography, pain, fatigue, impulsive behavior disorders

\section{INTRODUCTION}

Parkinson's disease (PD) is a common and devastating, progressive movement disorder. Its hallmark pathology is the loss of dopaminergic neurons in the substantia nigra, causing the key motor symptoms of tremor, rigidity, and bradykinesia. However, due to its multisystem character the disease manifests with various non-motor symptoms (NMS), such as hyposmia, depression, cognitive decline, and psychosis, autonomic disturbances, and sleep disturbances. Advanced neuroimaging methods render the view toward understanding the neural basis of these NMS in PD, as they allow a "window" into the underlying pathophysiological abnormalities. This review provides an up-to-date description of structural and functional alterations assessed by magnetic resonance imaging (MRI), positron emission tomography (PET), and functional magnetic resonance imaging (fMRI) underlying distinct NMS in the brain of PD patients.

Structural changes can be studied by using advanced MRI methods, such as voxel-based morphometry (VBM), diffusion tensor imaging (DTI), or susceptibility weighted imaging (SWI). The VBM is based on 3D-T1-weighted images and compares changes of gray and white matter volume among groups. DTI and fiber tracking are useful tools to study the three-dimensional diffusion of water as a function of spatial location and to display microstructural changes of white matter. The diffusion tensor may be used to characterize the magnitude, anisotropy, and orientation of the diffusion tensor. SWI is particularly sensitive to compounds which distort the local magnetic field, such as venous blood, hemorrhage, iron, and calcium. All these methods can be applied without any a priori assumptions (whole-brain approach) or with respect to a region of interest. The fMRI measures brain activity by detecting changes associated with blood flow because cerebral blood flow and neuronal activation are coupled [blood oxygen level-dependent contrast imaging (BOLD)]. The resting-state fMRI (rs-fMRI) evaluates regional interactions that occur when a subject is not performing an explicit task. The resting-state functional connectivity technique investigates the correlation patterns of BOLD signals between regions of interest and other brain regions. There are several anatomically separated brain regions that are functionally linked during rest: the default 
mode network (DMN), the sensorimotor, visual, executive (mainly dorsolateral prefrontal cortex and anterior cingulate cortex), salience (insular), frontal, parietal, auditory, and temporal networks (Figure 1). Functional communication between these brain regions plays a pivotal role in complex cognitive processes. Thus, the examination of functional connectivity provides insights in the core organization of the brain. There are several methods to analyze rs-fMRI data. Overall, one can distinguish between model-dependent and model-free methods. Model-dependent methods, including seed methods, correlate the data of a specific brain region (the "seed") against the timeseries of other regions. Model-free methods include for instance the principal component analysis or independent component analysis. Here, connectivity patterns were analyzed without the need of defining an a priori seed region (1).

An important issue in analyzing structural and functional MRI data is the specification of an appropriate threshold for statistical maps. Running the statistical analysis separately for each voxel creates the problem of multiple comparisons which increases the risk of false-positive results. Therefore, especially

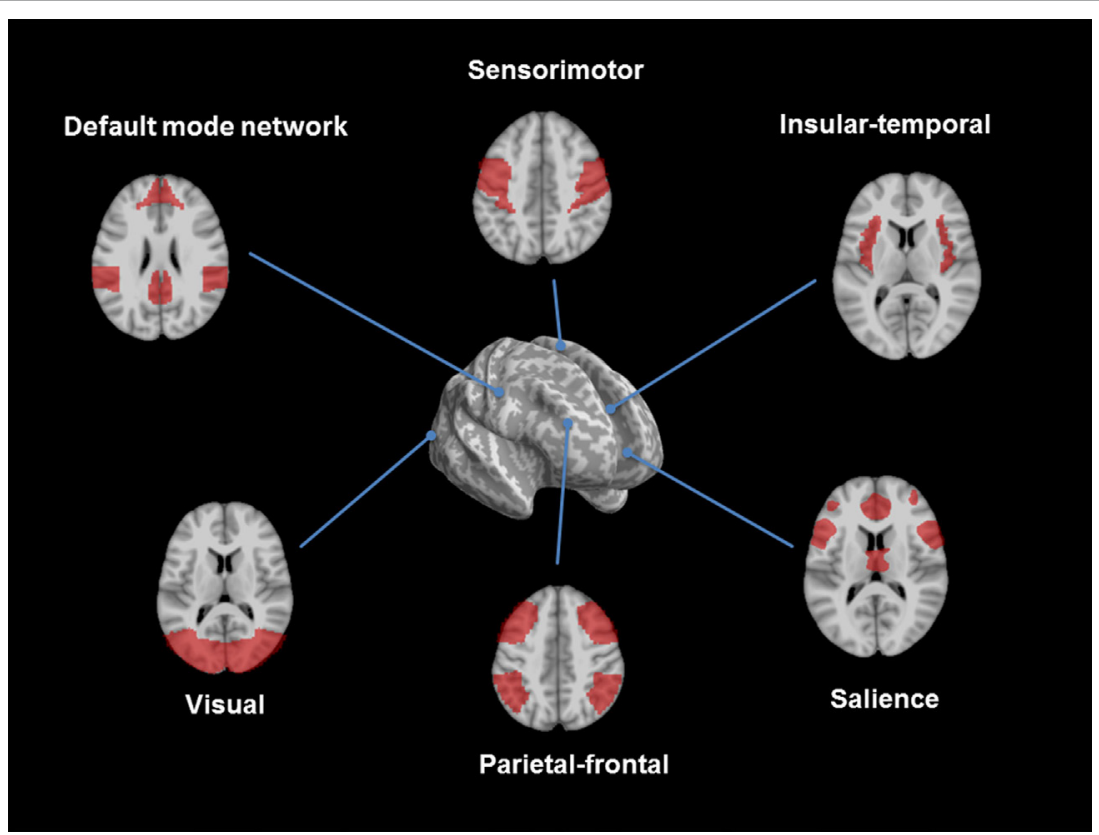

FIGURE 1 | Overview of resting-state networks. The figure shows the consistent reported resting-state networks: the default mode network, the sensorimotor, the insular/temporal and anterior cingulate cortex regions, the salience, the executive control, and the visual network (figures developed with SPM 8, www. fil.ion. ucl.ac.uk/spm)

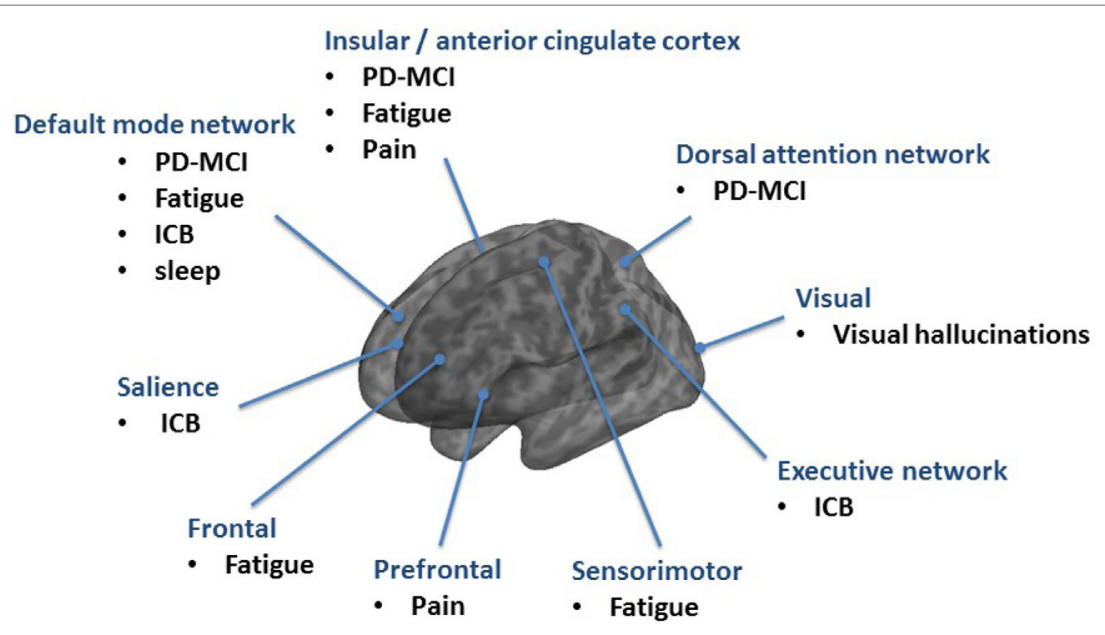

FIGURE 2 | Overview of resting-state networks mainly involved in non-motor syndromes in Parkinson's disease (PD). 
whole-brain and model-free analyses should incorporate a correction for multiple comparisons, such as the family-wise error method. The PET is a nuclear functional imaging technique that detects pairs of gamma rays emitted indirectly by a positronemitting radionuclide (tracer). Depending on the research or clinical questions several tracers are available [e.g., fluorine-18 (F-18) fluorodeoxyglucose (FDG)].

\section{SEARCH STRATEGIES AND SELECTION CRITERIA}

PubMed database was searched for articles on neuroimaging studies in PD by using a number of terms and combinations ("Parkinson," "functional imaging," "PET," "MRI," "non-motor," "fatigue," "hallucination," "dementia," "cognition," "pain," “impulsive compulsive behavior," "impulse control disorders," "sleep disorders," "RBD"). Articles were restricted to those: (1) in English and (2) published between 2000 and November, 2017. All abstracts were screened for relevance and the most pertinent articles were reviewed in full, with further examination of the corresponding reference lists. Since imaging of apathy, anxiety, and depression were recently reviewed these NMS were excluded (2).

\section{COGNITION}

Cognitive deficits are common in PD and can be present even as mild dysfunction in the prodromal and early stages or as dementia (PDD) in advanced stages (3). Cognitive function in PD deteriorates over time. $24 \%$ of the PD patients have cognitive disturbances at onset of the disease and every second patient shows progressive cognitive decline in the first 3 years $(4,5)$. In the long-term follow-up, the cumulative prevalence rates of PDD increase up to $80 \%$. Therefore, it seems that, regardless of the time of PD onset, the evolution of PDD occurs at around 70 years of age, and affects cognitive domains in a similar way $(6,7)$. There is still discussion about the role and localization of mild cognitive impairment in $\mathrm{PD}$ (PD-MCI) in between the spectrum of cognitive function and dysfunction. Therefore, the Movement Disorders Society Study group developed PD-MCI criteria (8). PD-MCI is characterized by a decreased prefrontal, temporal and parietal metabolism as well as an increase in brainstem/ cerebellar metabolism in FDG-PET $(9,10)$. It seems that with ongoing cognitive decline in PDD this hypometabolism spreads to the anterior cingulate cortex (10). However, the analysis of only 13 patients with PDD and the missing correction for multiple comparisons in the study by Yong et al. limits the generalizability of this result (10). The anterior cingulate cortex plays a role in a wide variety of autonomic functions and certain higher-level functions, such as decision-making, impulse control, and error detection which explains typical clinical problems in PDD. A recent $\mathrm{fMRI}$ study shows that a hypoactivation in the anterior cingulate cortex can also be observed in early and non-demented PD which could probably explain the impaired ability to shift attention between stimuli (i.e., shifting attentional "set") as part of the dysexecutive syndrome in PD (11). A complex network analysis approach revealed that PD-MCI is characterized by both, increments of local interconnectedness and connectivity decrements predominantly affecting long-range connections (12). Of notice, hyperconnectivity was demonstrated in PD patients without cognitive deficits, suggesting a recruitment of additional resource areas as initial response to progressive cell loss (13). However, with ongoing cognitive decline hyoconnectivites and disruptions of networks occur. In particular, the DMN emerged as a key function for cognitive deficits in PD (14). A decreased functional connectivity of the medial temporal lobe and inferior parietal cortex was found within the DMN (15). Using fMRI, a dysfunction of DMN during an executive task was detected in PD (16) and it was shown that the posterior cingulate cortex as major node within the DMN is linked to cognitive impairment in PD $(17,18)$. Using the posterior cingulate cortex as a seed for analysis, a significant decrease of connectivity was found in PD-MCI in the bilateral prefrontal cortex, left parieto-occipital junction, and right temporal gyrus and in PDD in the right inferior frontal gyrus as compared to non-demented PD (19, 20). A model-free approach using independent component analysis revealed that the connectivity between the dorsal attention network and right anterior insula with its adjacent frontal areas is reduced in PD-MCI and increased between posterior cortical regions and the DMN (21). The anterior insula is, therefore, critical for switching between dorsal attention network and DMN in the resting state and across different tasks $(21,22)$ (Figure 2).

Positron emission tomography studies using $\left[{ }^{11} \mathrm{C}\right]-\mathrm{MP} 4 \mathrm{~A}$ showed a significantly reduced cortical acetylcholinesterase activity in PDD and PD subjects when compared with healthy control subjects (23). The reduced cortical binding of $\left[{ }^{11} \mathrm{C}\right]-\mathrm{MP} 4 \mathrm{~A}$ with increasing signal diminution from frontal to occipital regions in PDD was comparable to the pattern in dementia with Lewy bodies (24). Whereas, in Alzheimer's disease the reduction of $\left[{ }^{11} \mathrm{C}\right]-\mathrm{MP} 4 \mathrm{~A}$ binding is restricted to the hippocampus, the temporoparietal cortex, and amygdala which underlines that PDD relies on an extensive cholinergic denervation $(25,26)$.

In terms of structural changes, there are conflicting results in PD, PD-MCI, and PDD. One study observed gray matter loss in cortical and subcortical regions of the prefrontal, temporal, and parieto-occipital cortex in non-demented PD patients in comparison to healthy controls, while another did not $(27,28)$. A meta-analysis of gray matter volume differences between patients with PD and healthy controls mainly found that patients with PD have regional gray matter volume reductions in the left inferior frontal gyrus extending to the superior temporal gyrus and the insula (29). This is of interest, since the left inferior frontal gyrus is specifically associated with cognitive processes and is involved in processing the motivational or emotional value of incoming information (29). Studies investigating gray matter atrophy in PD-MCI were negative or observed gray matter atrophy in the frontal, hippocampal, temporal, and parieto-occipital regions (28, 30-38). Structural changes in frontal and limbic system in PD-MCI were found to be associated with impaired performance in the Mini-Mental State Examination score, whereas frontal lobe atrophy was found to be associated with low performance in the Montreal Cognitive Assessment score, suggesting that atrophy of limbic 
lobes is associated with impaired memory, whereas frontal lobe atrophy is associated with executive dysfunction (39). The conflicting results in terms of PD-MCI may reflect the limitations of VBM to track subtle cortical atrophy in early stages of the disease, because cortical thinning assessed by surfaced-based analyses was consistently observed in frontal, temporal, and parietal regions in PD-MCI (40-43). While the extent of gray matter atrophy in non-demented PD and PD-MCI is a topic of contentious debate, the gray matter atrophy is well established in PDD. Subsequently, with disease progression and onset of PDD, diffuse gray matter loss becomes evident bilaterally in the hippocampus and parahippocampal gyrus, and in the occipital lobe, the frontal and parietal lobe, as well as some subcortical regions $(31,44)$.

Beside gray matter atrophy, the analysis of whiter matter with DTI revealed widespread changes in PD patients with cognitive dysfunction. Relative to healthy controls and non-demented PD patients, PD-MCI patients showed widespread white matter abnormalities in the cingulum, anterior and superior corona radiata, genu, and body of the corpus callosum, and inferior fronto-occipital, uncinate, and superior longitudinal fasciculi $(37,45,46)$. The loss of microstructural white matter integrity in PD increases with cognitive dysfunction (45). These DTI results indicate that central white matter tracts degenerate early in PD and that cognitive dysfunction in PD-MCI is linked to axonal damage (46).

These results have expanded the understanding of cognitive impairment in PD beyond fronto-striatal circuit dopaminergic deficits. Structural MRI studies have revealed gray matter atrophy and disruptions of white matter integrity in PDD, although findings in non-demented PD and PD-MCI remain inconstant. The lack of reproducibility in PD-MCI and non-demented PD can be explained by the heterogeneity of subjects, low sample size in some studies, different types of analysis in terms of methods, MRI parameters, or template choice. Furthermore, there are opposing views on PD-MCI itself and the neuropsychological assessments differ widely between the studies. In addition, there is a large heterogeneity across studies in terms of clinical stage, medication, and presence of other NMS. In particular, there is a need for multicenter longitudinal studies to clarify the spatial and temporal progression of morphological changes in cognitive decline in PD.

\section{FATIGUE}

Fatigue is one of the most common and disabling NMS in PD (47-49). Fatigue is a leading cause of disability and dramatically impairs quality of life $(50,51)$. Although a lack of consensus exists regarding a precise definition of $\mathrm{PD}$ fatigue, the core of fatigue is a "feeling of abnormal and overwhelming tiredness and lack of energy, distinct both qualitatively and quantitatively from normal tiredness" [cited from Ref. (52), p. 54]. Despite its enormous impact and high prevalence, little progress has been made in understanding the etiology or pathophysiology of fatigue.

The rs-fMRI demonstrates that brain areas, including frontal, postcentral, and anterior cingulate cortex regions, are involved in fatigue in PD patients (53). In another rs-fMRI study the amplitude of low-frequency fluctuations (ALFF) was used to measure regional brain activity and functional connectivity was investigated at a network level. PD-related fatigue was associated with ALFF changes in the attention network (right middle frontal gyrus) and in the salience network (left insula, right midcingulate cortex). Corresponding to that, functional connectivity was altered mainly in the temporal, parietal, and motor cortices (54). The study by Tessitore et al. studied a cohort of "drug-naïve" patients with PD with rs-fMRI and independent component analysis as model-free approach $(55,56)$. Fatigue was associated with a decreased connectivity in the sensorimotor network (supplementary motor area) and an increased connectivity in the DMN (prefrontal and posterior cingulate cortices). Fatigue severity, as assessed with the 16-item Parkinson fatigue scale was correlated with both sensorimotor and DMN connectivity changes $(55,56)$. The involvement of the frontal lobe in PD-fatigue was also demonstrated with SPECT (99mTc-HMPAO) showing a significant correlation between the fatigue scale and the reduction of perfusion in the frontal lobe in patients with PD (57). In a recent FDG-PET study high Fatigue Severity Scale scores were associated with brain hypermetabolism in areas, including the right middle temporal gyrus (Brodmann area 37) and left middle occipital gyrus (Brodmann area 19), and hypometabolism in regions, such as the right precuneus (Brodmann area 23), left inferior frontal gyrus (Brodmann area 45), and left superior frontal gyrus (orbital part, Brodmann area 11) (54). The FDG-PET revealed that metabolic changes in cortical regions associated with the salience (i.e., right insular region) and default (i.e., bilateral posterior cingulate cortex) networks display a significant correlation with the level of fatigue (58). In terms of structural changes, there were no significant volume differences of gray matter between PD with and without fatigue in the VBM analysis $(55,56)$. In summary, multiple brain areas underlie fatigue, including frontal, temporal, and parietal regions indicative of emotion, motivation, and cognitive functions (Figure 2).

\section{VISUAL HALLUCINATIONS}

Visual hallucinations are the most common manifestation of psychosis in PD and are predictive for a rapid cognitive decline. VBM studies have reported gray matter atrophy in multiple regions, but, overall, the results remain inconsistent. Gray matter atrophy was observed in areas related to visuospatial processing, attention, and memory, suggesting visual hallucinations are the correlate of dysfunctions in multiple cortical as well as subcortical brain regions. The involved areas include the primary visual cortex, visual association cortex, limbic and para-limbic regions, the pedunculopontine nucleus, and substantia innominate (59-65).

Also white matter changes were found when visual hallucinations are present. The mean diffusivity was increased in the parieto-temporal region in the non-demented $\mathrm{PD}$ patients with visual hallucinations and increased diffusely in the presence of dementia, including the fronto-occipital regions (66). Functional imaging studies consistently revealed the involvement of visual pathways in the pathogenesis of visual hallucinations, including alterations in both dorsal and ventral visual pathways (67-74). 
It was hypothesized that PD patients with visual hallucinations have a reduced responsiveness to external visual stimuli (bottom-up) and an abnormally increased frontal activation (top-down) $(67,75)$. Regional cerebral blow flow, assessed with SPECT and N-isopropyl-p-[(123)I]iodoamphetamine, showed a hypoperfusion of the right fusiform gyrus (visual recognition) and an hyperperfusion of the right superior and temporal gyri (generation of complex visual images) in PD patients with visual hallucinations (75). Also the serotonergic system seems to play a role in visual hallucinations. Patients having PD with visual hallucinations demonstrate increased serotonin $2 \mathrm{~A}$ receptor binding (PET with $\left[{ }^{18} \mathrm{~F}\right]$-Setoperone) in the ventral visual pathway (including the bilateral inferooccipital gyrus, right fusiform gyrus, and inferotemporal cortex) as well as the bilateral dorsolateral prefrontal cortex, medial orbitofrontal cortex, and insula (76).

\section{IMPULSE CONTROL BEHAVIOR}

Impulsive control behaviors (ICB) include gambling disorder, binge eating disorder, compulsive sexual behavior, and compulsive shopping and occur in $17 \%$ of patients treated with a dopamine agonist. These behaviors probably reflect the interactions of the individual's susceptibility and the dopaminergic medicationsin PD (77). There is some evidence that some patients are prone to develop ICB. In general, PD-ICB has been associated mainly with brain alterations involving the fronto-striatal and fronto-limbic circuits. In a recent study in $\mathrm{PD}$ patients with and without $\mathrm{ICB}$, the presence of ICB symptoms was associated with an increased connectivity within the salience network and DMN, as well as with a decreased connectivity within the central executive network (78). Drug-naïve PD patients who develop ICB in the follow-up showed at baseline a decreased connectivity in the DMN and executive network and increased connectivity in the salience network, suggesting that these cognitive and limbic connectivity changes are predictive for the development of ICB in PD (79). The involvement of the dopaminergic system in the pathophysiology of ICB has been demonstrated by several functional imaging studies showing an abnormal sensitization of the dopaminergic system. In line with the sensitization theory PET studies using $\left[{ }^{11} \mathrm{C}\right]$-Raclopride have shown a greater striatal dopamine release after levodopa intake in PD patients with ICD. In contrast to PD without ICB the PD patients with dopamine dysregulation syndrome exhibited enhanced levodopa-induced ventral striatal dopamine release and this sensitized striatal dopamine transmission correlated with self-reported compulsive drug "wanting" but not "liking" (80). Also a reduced $\left[{ }^{11} \mathrm{C}\right]$-Raclopride binding in the ventral striatum of PD patients with pathological gambling likely reflects greater dopaminergic release (81). Interestingly that greater striatal dopamine release and abnormal sensitization of the dopaminergic system after a levodopa dose intake was only observed following reward-related cue exposure, relative to neutral cue exposure (82). This heightened response of striatal reward circuitry to heterogeneous reward-related visual cues was observed among a group of patients with different kind of ICBs. Therefore, one can assume that in vulnerable individuals there is some kind of global sensitization to appetitive behaviors with dopaminergic therapy (82). In a fMRI study of PD patients with hypersexuality the exposure to sexual cues significantly increased sexual desire and this was accompanied by significant signal changes in regions corresponding to emotional, cognitive, autonomic, visual, and motivational processes (limbic, paralimbic, temporal, occipital, somatosensory, and prefrontal cortices). Moreover, the increased sexual desire correlated with enhanced activations in the ventral striatum, and cingulate and orbitofrontal cortices (83). When the PD patients with hypersexuality were OFF medication, the activation during the presentation of sexual cues decreased. However, this decrease was not present when the patients were $\mathrm{ON}$ medication. One can, therefore, hypothesize that dopamine drugs may release inhibition within local cortical neuronal circuits (83). In summary, a consistent feature of ICB is the increased cue reactivity in the striatum. Using a pharmacological manipulation and a risk taking task while performing fMRI PD patients with ICB made more risky choices. This was accompanied with decreased activity in the orbitofrontal cortex and anterior cingulate cortex in comparison to PD controls (84). PD patients with ICB appear to have a bias toward risky choices and dopamine agonists seem to enhance the sensitivity to risk because they impair the striatal risk evaluation (84).

Furthermore, PD patients with pathological gambling showed resting-state overactivity in the orbitofrontal cortex, the hippocampus, the amygdala, the insula, and the ventral pallidum (mesocorticolimbic network) (85). The mesocorticolimbic dopamine network guides reward-motivated behavior; however, its role in PD-ICB is not yet understood. A recent fMRI study showed elevated ventral striatal connectivity to the anterior cingulate gyrus, orbitofrontal cortex, insula, putamen, globus pallidus, and thalamus in PD patients with ICB, suggesting that PD-ICB patients have elevated network connectivity in the mesocorticolimbic network. Behaviorally, proficient reward-based learning is related to this enhanced limbic and ventral striatal connectivity (86) (Figure 2).

In terms of gray matter changes, the studies in PD-ICB are inconsistent $(55,56,87,88)$. One study aimed to answer the issue mentioned above whether de novo $\mathrm{PD}$ patient who develop ICB have specific structural abnormalities. Patients who went on to develop ICB did not show significant structural changes in comparison to PD patients without ICB (89). Reduced cortical thickness of fronto-striatal regions but also increased volume of amygdala and a positive correlation between ICB severity and fronto-parietal gray matter volumes has been reported $(90,91)$. In terms of white matter structural changes, an increased fractional anisotropy of the genu of the anterior corpus callosum, and right internal capsule, right posterior cingulum, and right thalamic radiations was observed in PD-ICB (92). A recent comprehensive study using DTI, rs-fMRI, and surface-based morphometry provided a broad picture of structural and functional alterations in PD-ICB patients. Compared with PD without ICB, patients with PD-ICB were characterized by precentral and superior frontal cortical thinning, and motor and extramotor white matter 
tract damage. Relative to $\mathrm{PD}$ without ICB, PD-ICB patients were characterized by a more severe involvement of frontal, meso-limbic, and motor circuits. ICB in PD is then probably the result of a disconnection between sensorimotor, associative, and cognitive networks (93).

\section{SLEEP DISORDERS}

Sleep disorders in PD are common and include heterogeneous group of symptoms and reasons, such as nightly motor symptoms, REM sleep behavior disorders (RBD), or daytime sleepiness. $\mathrm{RBD}$ is a parasomnia with dream enactment and violent limb moves, which can occur in the prodromal phase of PD. Sleep disorders were found to be related to nigrostriatal dopaminergic degeneration and around $20-40 \%$ of RBD patients in the prodromal state of PD show deficits in the dopamine transporters $(94,95)$. The results in terms of brain perfusion and metabolism in prodromal cohorts, however, remain inconsistent [recently reviewed in Ref. $(96,97)$ ]. Since sleep can have a restorative role in the brain a major question in PD is the link between sleep disturbances and cognitive deficits. PD patients with sleep disturbance showed poorer performance in attention/working memory and were characterized by a more extensive cortical thinning in the left fronto-parietal regions and white matter disintegration in widespread regions when compared to those without sleep disturbance (98). Cortical functional connectivity in PD patients with sleep disturbance with a seed in the DMN and dorsal attention network exhibited a less severe decrease when compared to those without sleep disturbance. These data suggest that sleep disturbances are associated with white matter and alterations in functional networks in conjunction with cognitive impairment (98). In PD patients with RBD, whiter matter alterations were also observed in the cingulum and left inferior occipital fasciculus and might explain faster cognitive decline in terms of visual recognition and visuospatial dysfunction and executive function in these patients $(99,100)$. This is in line with the fact that RBD is a predictor of PDD.

\section{PAIN}

Pain is a frequent symptom in PD with great impact on mobility and quality of life. Pain can occur in both premotor and motor stages and may be linked to musculoskeletal, dystonic, radicular, neuropathic and central causes $(101,102)$. The so called "pain matrix" as a fluid system composed of several interacting networks encompasses several brain areas which are involved in pain processing $(103,104)$. The main components of this network in terms of acute pain are: primary and secondary somatosensory, insular, anterior cingulate, prefrontal cortices, and thalamus (105). However, pain is not only nociception because many factors (e.g., cognition, depression, and social status) can separately influence pain perception. Nociceptive inputs can activate complex interactions among central sites, including cortical regions that are active in cognitive, emotional, and reward functions (106). Therefore, multiple other areas were found to be related to pain processing, such as basal ganglia, brainstem, cerebellum, and hippocampus $(103,106,107)$. With ongoing pain or chronic pain, there is a continuous reorganization of the cortex (108). The pain threshold in PD patients was found to be lower than in healthy controls and the administration of levodopa significantly raised the pain threshold in PD but not in controls. With the reduced pain threshold, there was a significant increase in pain-induced activation in the painmatrix, namely right insula and prefrontal and left anterior cingulate cortices in PD compared to control group. Moreover, levodopa significantly reduced this pain-induced cortical activation and increases pain threshold, providing evidence for the involvement of the dopaminergic system in the modulation of pain in PS $(109,110)$. In early, "drug-naive" PD patients not experiencing pain symptoms, the event-related fMRI suggest that a functional remodulation of pain processing pathways occurs even in the absence of clinically overt pain symptoms. A greater activation of the left somatosensory cortex, left cerebellum and right low pons (in an area encompassing the nucleus rafe magnus and the gigantocellular/paragigantocellular nuclei) during noxious stimulations is present in "drug-naive" PD patients when compared to healthy controls (111). In summary, these data suggest that a compensatory reorganization of pain-related brain areas, induced by early neuropathological changes, is present in "drug-naive" PD patients, not reporting pain symptoms. These mechanisms may then become dysfunctional during disease course and impact pain-threshold and contribute to the emergence of pain symptoms in more advanced PD stages (111).

\section{CONCLUSION}

The multisystem character of PD and its clinical heterogeneity in disease presentation and progression challenges the understanding of NMS in PD, especially at early stages. To better understand the nature and pathophysiology of NMS multiple biomarkers derived from different imaging modalities seems to be the appropriate way. Moreover, ideally these data should be linked to other biomarkers, e.g., derived from cerebrospinal fluid in order improve our understanding and to weight the importance of imaging findings. Further research is needed to perform multicenter studies, to improve measurement techniques, and to standardize research protocols. An important issue in PD is that patients usually have a large panel of different motor and NMS with each having impact on the individual brain signatures. Therefore, it is of importance to assess as many as possible cofounders with valid and reliable methods before analyzing imaging data. This is of importance, because future clinical decision-making and treatment may likely rely upon in vivo imaging.

\section{AUTHOR CONTRIBUTIONS}

Developing and writing: TP. 


\section{REFERENCES}

1. van den Heuvel MP, Hulshoff Pol HE. Exploring the brain network: a review on resting-state fMRI functional connectivity. Eur Neuropsychopharmacol (2010) 20(8):519-34. doi:10.1016/j.euroneuro.2010.03.008

2. Thobois S, Prange S, Sgambato-Faure V, Tremblay L, Broussolle E. Imaging the etiology of apathy, anxiety, and depression in Parkinson's disease: implication for treatment. Curr Neurol Neurosci Rep (2017) 17(10):76. doi:10.1007/s11910-017-0788-0

3. Fengler S, Liepelt-Scarfone I, Brockmann K, Schäffer E, Berg D, Kalbe E. Cognitive changes in prodromal Parkinson's disease: a review. Mov Disord (2017) 32(12):1655-66. doi:10.1002/mds.27135

4. Muslimovic D, Post B, Speelman JD, Schmand B. Cognitive profile of patients with newly diagnosed Parkinson disease. Neurology (2005) 65:1239-45. doi:10.1212/01.wnl.0000180516.69442.95

5. Muslimovic D, Post B, Speelman JD, de Haan RJ, Schmand B. Cognitive decline in Parkinson's disease: a prospective longitudinal study. J Int Neuropsychol Soc (2009) 15:426-37. doi:10.1017/S1355617709090614

6. Reid WG, Hely MA, Morris JG, Loy C, Halliday GM. Dementia in Parkinson's disease: a 20-year neuropsychological study (Sydney multicentre study). J Neurol Neurosurg Psychiatry (2011) 82:1033-7. doi:10.1136/jnnp. 2010.232678

7. Aarsland D, Andersen K, Larsen JP, Lolk A, Kragh-Sørensen P. Prevalence and characteristics of dementia in Parkinson disease: an 8-year prospective study. Arch Neurol (2003) 60(3):387-92. doi:10.1001/archneur.60.3.387

8. Litvan I, Goldman JG, Tröster AI, Schmand BA, Weintraub D, Petersen RC, et al. Diagnostic criteria for mild cognitive impairment in Parkinson's disease: movement disorder society task force guidelines. Mov Disord (2012) 27(3):349-56. doi:10.1002/mds.24893

9. Huang C, Mattis P, Perrine K, Brown N, Dhawan V, Eidelberg D. Metabolic abnormalities associated with mild cognitive impairment in Parkinson disease. Neurology (2008) 70(16 Pt 2):1470-7. doi:10.1212/01. wnl.0000304050.05332.9c

10. Yong SW, Yoon JK, An YS, Lee PH. A comparison of cerebral glucose metabolism in Parkinson's disease, Parkinson's disease dementia and dementia with Lewy bodies. Eur J Neurol (2007) 14(12):1357-62.

11. Gruszka A, Hampshire A, Barker RA, Owen AM. Normal aging and Parkinson's disease are associated with the functional decline of distinct frontal-striatal circuits. Cortex (2017) 93:178-92. doi:10.1016/j.cortex.2017. 05.020

12. Baggio HC, Sala-Llonch R, Segura B, Marti MJ, Valldeoriola F, Compta Y, et al. Functional brain networks and cognitive deficits in Parkinson's disease. Hum Brain Mapp (2014) 35(9):4620-34. doi:10.1002/hbm.22499

13. Gorges M, Müller HP, Lulé D; Landscape Consortium, Pinkhardt EH, Ludolph AC, et al. To rise and to fall: functional connectivity in cognitively normal and cognitively impaired patients with Parkinson's disease. Neurobiol Aging (2015) 36(4):1727-35. doi:10.1016/j.neurobiolaging. 2014.12.026

14. Tahmasian M, Eickhoff SB, Giehl K, Schwartz F, Herz DM, Drzezga A, et al. Resting-state functional reorganization in Parkinson's disease: an activation likelihood estimation meta-analysis. Cortex (2017) 92:119-38. doi:10.1016/j.cortex.2017.03.016

15. Tessitore A, Esposito F, Vitale C, Santangelo G, Amboni M, Russo A, et al. Default-mode network connectivity in cognitively unimpaired patients with Parkinson disease. Neurology (2012) 79(23):2226-32. doi:10.1212/ WNL.0b013e31827689d6

16. van Eimeren T, Monchi O, Ballanger B, Strafella AP. Dysfunction of the default mode network in Parkinson disease: a functional magnetic resonance imaging study. Arch Neurol (2009) 66(7):877-83. doi:10.1001/ archneurol.2009.97

17. Fransson P, Marrelec G. The precuneus/posterior cingulate cortex plays a pivotal role in the default mode network: evidence from a partial correlation network analysis. Neuroimage (2008) 42(3):1178-84. doi:10.1016/j. neuroimage.2008.05.059

18. Mattila PM, Rinne JO, Helenius H, Dickson DW, Röyttä M. Alpha-synucleinimmunoreactive cortical Lewy bodies are associated with cognitive impairment in Parkinson's disease. Acta Neuropathol (2000) 100(3):285-90. doi:10.1007/s004019900168
19. Rektorova I, Krajcovicova L, Marecek R, Mikl M. Default mode network and extrastriate visual resting state network in patients with Parkinson's disease dementia. Neurodegener Dis (2012) 10(1-4):232-7. doi:10.1159/ 000334765

20. Chen B, Wang S, Sun W, Shang X, Liu H, Liu G, et al. Functional and structural changes in gray matter of Parkinson's disease patients with mild cognitive impairment. Eur J Radiol (2017) 93:16-23. doi:10.1016/j.ejrad.2017.05.018

21. Baggio HC, Segura B, Sala-Llonch R, Marti MJ, Valldeoriola F, Compta Y, et al. Cognitive impairment and resting-state network connectivity in Parkinson's disease. Hum Brain Mapp (2015) 36(1):199-212. doi:10.1002/ hbm. 22622

22. Sridharan D, Levitin DJ, Menon V. A critical role for the right frontoinsular cortex in switching between central-executive and default-mode networks. Proc Natl Acad Sci U S A (2008) 105(34):12569-74. doi:10.1073/ pnas.0800005105

23. Bohnen NI, Kaufer DI, Hendrickson R, Ivanco LS, Lopresti BJ, Constantine GM, et al. Cognitive correlates of cortical cholinergic denervation in Parkinson's disease and parkinsonian dementia. J Neurol (2006) 253(2):242-7. doi:10.1007/s00415-005-0971-0

24. Klein JC, Eggers C, Kalbe E, Weisenbach S, Hohmann C, Vollmar S, et al. Neurotransmitter changes in dementia with Lewy bodies and Parkinson disease dementia in vivo. Neurology (2010) 74(11):885-92. doi:10.1212/ WNL.0b013e3181d55f61

25. Shinotoh H, Namba H, Fukushi K, Nagatsuka S, Tanaka N, Aotsuka A, et al. Progressive loss of cortical acetylcholinesterase activity in association with cognitive decline in Alzheimer's disease: a positron emission tomography study. Ann Neurol (2000) 48(2):194-200. doi:10.1002/1531-8249(200008) 48:2<194::AID-ANA9>3.0.CO;2-X

26. Bohnen NI, Kaufer DI, Ivanco LS, Lopresti B, Koeppe RA, Davis JG, et al. Cortical cholinergic function is more severely affected in parkinsonian dementia than in Alzheimer disease: an in vivo positron emission tomographic study. Arch Neurol (2003) 60(12):1745-8. doi:10.1001/archneur. 60.12.1745

27. Lee EY, Sen S, Eslinger PJ, Wagner D, Shaffer ML, Kong L, et al. Early cortical gray matter loss and cognitive correlates in non-demented Parkinson's patients. Parkinsonism Relat Disord (2013) 19(12):1088-93. doi:10.1016/j. parkreldis.2013.07.018

28. Weintraub D, Doshi J, Koka D, Davatzikos C, Siderowf AD, Duda JE, et al. Neurodegeneration across stages of cognitive decline in Parkinson disease. Arch Neurol (2011) 68(12):1562-8. doi:10.1001/archneurol.2011.725

29. Pan PL, Song W, Shang HF. Voxel-wise meta-analysis of gray matter abnormalities in idiopathic Parkinson's disease. Eur J Neur (2012) 19(2):199-206. doi:10.1111/j.1468-1331.2011.03474.x

30. Summerfield C, Junqué C, Tolosa E, Salgado-Pineda P, Gómez-Ansón B, Martí MJ, et al. Structural brain changes in Parkinson disease with dementia: a voxel-based morphometry study. Arch Neurol (2005) 62(2):281-5. doi:10.1001/archneur.62.2.281

31. Melzer TR, Watts R, MacAskill MR, Pitcher TL, Livingston L, Keenan RJ, et al. Grey matter atrophy in cognitively impaired Parkinson's disease. J Neurol Neurosurg Psychiatry (2012) 83(2):188-94. doi:10.1136/jnnp-2011300828

32. Chen FX, Kang DZ, Chen FY, Liu Y, Wu G, Li X, et al. Gray matter atrophy associated with mild cognitive impairment in Parkinson's disease. Neurosci Lett (2016) 617:160-5. doi:10.1016/j.neulet.2015.12.055

33. Noh SW, Han YH, Mun CW, Chung EJ, Kim EG, Ji KH, et al. Analysis among cognitive profiles and gray matter volume in newly diagnosed Parkinson's disease with mild cognitive impairment. J Neurol Sci (2014) 347(1-2):210-3. doi:10.1016/j.jns.2014.09.049

34. Beyer MK, Janvin CC, Larsen JP, Aarsland D. A magnetic resonance imaging study of patients with Parkinson's disease with mild cognitive impairment and dementia using voxel-based morphometry. J Neurol Neurosurg Psychiatry (2007) 78(3):254-9. doi:10.1136/jnnp.2006.093849

35. Dalaker TO, Zivadinov R, Larsen JP, Beyer MK, Cox JL, Alves G, et al. Gray matter correlations of cognition in incident Parkinson's disease. Mov Disord (2010) 25(5):629-33. doi:10.1002/mds.22867

36. Hattori T, Orimo S, Aoki S, Ito K, Abe O, Amano A, et al. Cognitive status correlates with white matter alteration in Parkinson's disease. Hum Brain Mapp (2012) 33(3):727-39. doi:10.1002/hbm.21245 
37. Agosta F, Canu E, Stefanova E, Sarro L, Tomić A, Špica V, et al. Mild cognitive impairment in Parkinson's disease is associated with a distributed pattern of brain white matter damage. Hum Brain Mapp (2014) 35(5):1921-9. doi:10.1002/hbm.22302

38. Amboni M, Tessitore A, Esposito F, Santangelo G, Picillo M, Vitale C, et al. Resting-state functional connectivity associated with mild cognitive impairment in Parkinson's disease. J Neurol (2015) 262(2):425-34. doi:10.1007/ s00415-014-7591-5

39. Gao Y, Nie K, Huang B, Mei M, Guo M, Xie S, et al. Changes of brain structure in Parkinson's disease patients with mild cognitive impairment analyzed via VBM technology. Neurosci Lett (2017) 658:121-32. doi:10.1016/j.neulet. 2017.08.028

40. Pagonabarraga J, Corcuera-Solano I, Vives-Gilabert Y, Llebaria G, GarcíaSánchez C, Pascual-Sedano B, et al. Pattern of regional cortical thinning associated with cognitive deterioration in Parkinson's disease. PLoS One (2013) 8(1):e54980. doi:10.1371/journal.pone.0054980

41. Pereira JB, Svenningsson P, Weintraub D, Brønnick K, Lebedev A, Westman E, et al. Initial cognitive decline is associated with cortical thinning in early Parkinson disease. Neurology (2014) 82(22):2017-25. doi:10.1212/WNL. 0000000000000483

42. Gerrits NJ, van Loenhoud AC, van den Berg SF, Berendse HW, Foncke EM, Klein M, et al. Cortical Thickness, surface area and subcortical volume differentially contribute to cognitive heterogeneity in Parkinson's disease. PLoS One (2016) 11(2):e0148852. doi:10.1371/journal.pone.0148852

43. Gasca-Salas C, García-Lorenzo D, Garcia-Garcia D, Clavero P, Obeso JA, Lehericy S, et al. Parkinson's disease with mild cognitive impairment: severe cortical thinning antedates dementia. Brain Imaging Behav (2017):1-9. doi:10.1007/s11682-017-9751-6

44. Burton EJ, McKeith IG, Burn DJ, Williams ED, O’Brien JT. Cerebral atrophy in Parkinson's disease with and without dementia: a comparison with Alzheimer's disease, dementia with Lewy bodies and controls. Brain (2004) 127(Pt 4):791-800. doi:10.1093/brain/awh088

45. Melzer TR, Watts R, MacAskill MR, Pitcher TL, Livingston L, Keenan RJ, et al. White matter microstructure deteriorates across cognitive stages in Parkinson disease. Neurology (2013) 80(20):1841-9. doi:10.1212/WNL. 0b013e3182929f62

46. Duncan GW, Firbank MJ, Yarnall AJ, Khoo TK, Brooks DJ, Barker RA, et al. Gray and white matter imaging: A biomarker for cognitive impairment in early Parkinson's disease? Mov Disord (2016) 31(1):103-10. doi:10.1002/ mds. 26312

47. Uebelacker LA, Epstein-Lubow G, Lewis T, Broughton MK, Friedman JH. A survey of Parkinson's disease patients: most bothersome symptoms and coping preferences. J Parkinsons Dis (2014) 4(4):717-23. doi:10.3233/JPD140446

48. Fu R, Luo XG, Ren Y, He ZY, Lv H. Clinical characteristics of fatigued Parkinson's patients and the response to dopaminergic treatment. Transl Neurodegener (2016) 5:9. doi:10.1186/s40035-016-0056-2

49. Zuo LJ, Yu SY, Wang F, Hu Y, Piao YS, Du Y, et al. Parkinson's disease with fatigue: clinical characteristics and potential mechanisms relevant to $\alpha$-synuclein oligomer. J Clin Neurol (2016) 12(2):172-80. doi:10.3988/ jcn.2016.12.2.172

50. Alves G, Wentzel-Larsen T, Larsen JP. Is fatigue an independent and persistent symptom in patients with Parkinson disease? Neurology (2004) 63(10):1908-11. doi:10.1212/01.WNL.0000144277.06917.CC

51. Stocchi F, Abbruzzese G, Ceravolo R, Cortelli P, D’Amelio M, De Pandis MF, et al. Prevalence of fatigue in Parkinson disease and its clinical correlates. Neurology (2014) 83(3):215-20. doi:10.1212/WNL.0000000000000587

52. Brown RG, Dittner A, Findley L, Wessely SC. The Parkinson fatigue scale. Parkinsonism Relat Disord (2005) 11(1):49-55. doi:10.1016/j.parkreldis. 2004.07.007

53. Li J, Yuan Y, Wang M, Zhang J, Zhang L, Jiang S, et al. Alterations in regional homogeneity of resting-state brain activity in fatigue of Parkinson's disease. J Neural Transm (2017) 124(10):1187-95. doi:10.1007/s00702-017-1748-1

54. Zhang JJ, Ding J, Li JY, Wang M, Yuan YS, Zhang L, et al. Abnormal resting-state neural activity and connectivity of fatigue in Parkinson's disease. CNS Neurosci Ther (2017) 23(3):241-7. doi:10.1111/cns. 12666

55. Tessitore A, Giordano A, De Micco R, Caiazzo G, Russo A, Cirillo M, et al. Functional connectivity underpinnings of fatigue in "drug-naïve" patients with Parkinson's disease. Mov Disord (2016) 31(10):1497-505. doi:10.1002/ mds. 26650
56. Tessitore A, Santangelo G, De Micco R, Vitale C, Giordano A, Raimo S, et al. Cortical thickness changes in patients with Parkinson's disease and impulse control disorders. Parkinsonism Relat Disord (2016) 24:119-25. doi:10.1016/j.parkreldis.2015.10.013

57. Abe K, Takanashi M, Yanagihara T. Fatigue in patients with Parkinson's disease. Behav Neurol (2000) 12(3):103-6.

58. Cho SS, Aminian K, Li C, Lang AE, Houle S, Strafella AP. Fatigue in Parkinson's disease: The contribution of cerebral metabolic changes. Hum Brain Mapp (2017) 38(1):283-92. doi:10.1002/hbm.23360

59. Ramírez-Ruiz B, Martí MJ, Tolosa E, Giménez M, Bargalló N, Valldeoriola F, et al. Cerebral atrophy in Parkinson's disease patients with visual hallucinations. EurJ Neurol (2007) 14(7):750-6. doi:10.1111/j.1468-1331.2007.01768.x

60. Ibarretxe-Bilbao N, Ramírez-Ruiz B, Tolosa E, Martí MJ, Valldeoriola F, Bargalló N, et al. Hippocampal head atrophy predominance in Parkinson's disease with hallucinations and with dementia. J Neurol (2008) 255(9): 1324-31. doi:10.1007/s00415-008-0885-8

61. Ibarretxe-Bilbao N, Ramirez-Ruiz B, Junque C, Marti MJ, Valldeoriola F, Bargallo N, et al. Differential progression of brain atrophy in Parkinson's disease with and without visual hallucinations. J Neurol Neurosurg Psychiatry (2010) 81(6):650-7. doi:10.1136/jnnp.2009.179655

62. Meppelink AM, de Jong BM, Teune LK, van Laar T. Regional cortical grey matter loss in Parkinson's disease without dementia is independent from visual hallucinations. Mov Disord (2011) 26(1):142-7. doi:10.1002/ mds. 23375

63. Janzen J, van 't Ent D, Lemstra AW, Berendse HW, Barkhof F, Foncke EM. The pedunculopontine nucleus is related to visual hallucinations in Parkinson's disease: preliminary results of a voxel-based morphometry study. J Neurol (2012) 259(1):147-54. doi:10.1007/s00415-011-6149-z

64. Watanabe H, Senda J, Kato S, Ito M, Atsuta N, Hara K, et al. Cortical and subcortical brain atrophy in Parkinson's disease with visual hallucination. Mov Disord (2013) 28(12):1732-6. doi:10.1002/mds.25641

65. Gama RL, Bruin VM, Távora DG, Duran FL, Bittencourt L, Tufik S. Structural brain abnormalities in patients with Parkinson's disease with visual hallucinations: a comparative voxel-based analysis. Brain $\operatorname{Cogn}(2014)$ 87:97-103. doi:10.1016/j.bandc.2014.03.011

66. Lee WW, Yoon EJ, Lee JY, Park SW, Kim YK. Visual hallucination and pattern of brain degeneration in Parkinson's disease. Neurodegener Dis (2017) 17(2-3):63-72. doi:10.1159/000448517

67. Stebbins GT, Goetz CG, Carrillo MC, Bangen KJ, Turner DA, Glover GH, et al. Altered cortical visual processing in PD with hallucinations: an fMRI study. Neurology (2004) 63(8):1409-16. doi:10.1212/01.WNL.0000141853. 27081.BD

68. Holroyd S, Wooten GF. Preliminary FMRI evidence of visual system dysfunction in Parkinson's disease patients with visual hallucinations. J Neuropsychiatry Clin Neurosci (2006) 18(3):402-4. doi:10.1176/jnp.2006. 18.3.402

69. Ramírez-Ruiz B, Martí MJ, Tolosa E, Falcón C, Bargalló N, Valldeoriola F, et al. Brain response to complex visual stimuli in Parkinson's patients with hallucinations: a functional magnetic resonance imaging study. Mov Disord (2008) 23(16):2335-43. doi:10.1002/mds.22258

70. Yao N, Shek-Kwan Chang R, Cheung C, Pang S, Lau KK, Suckling J, et al. The default mode network is disrupted in Parkinson's disease with visual hallucinations. Hum Brain Mapp (2014) 35(11):5658-66. doi:10.1002/ hbm. 22577

71. Nagano-Saito A, Washimi Y, Arahata Y, Iwai K, Kawatsu S, Ito K, et al. Visual hallucination in Parkinson's disease with FDG PET. Mov Disord (2004) 19(7):801-6. doi:10.1002/mds.20129

72. Boecker H, Ceballos-Baumann AO, Volk D, Conrad B, Forstl H, Haussermann P. Metabolic alterations in patients with Parkinson disease and visual hallucinations. Arch Neurol (2007) 64(7):984-8. doi:10.1001/archneur.64.7.984

73. Park HK, Kim JS, Im KC, Kim MJ, Lee JH, Lee MC, et al. Visual hallucinations and cognitive impairment in Parkinson's disease. Can J Neurol Sci (2013) 40(5):657-62. doi:10.1017/S0317167100014888

74. Matsui H, Nishinaka K, Oda M, Hara N, Komatsu K, Kubori T, et al. Hypoperfusion of the visual pathway in parkinsonian patients with visual hallucinations. Mov Disord (2006) 21(12):2140-4. doi:10.1002/mds.21147

75. Oishi N, Udaka F, Kameyama M, Sawamoto N, Hashikawa K, Fukuyama H. Regional cerebral blood flow in Parkinson disease with nonpsychotic visual hallucinations. Neurology (2005) 65(11):1708-15. doi:10.1212/01.wnl. 0000187116.13370.e0 
76. Ballanger B, Strafella AP, van Eimeren T, Zurowski M, Rusjan PM, Houle S, et al. Serotonin 2A receptors and visual hallucinations in Parkinson disease. Arch Neurol (2010) 67(4):416-21. doi:10.1001/archneurol.2010.35

77. Voon V, Napier TC, Frank MJ, Sgambato-Faure V, Grace AA, RodriguezOroz $\mathrm{M}$, et al. Impulse control disorders and levodopa-induced dyskinesias in Parkinson's disease: an update. Lancet Neurol (2017) 16(3):238-50. doi:10.1016/S1474-4422(17)30004-2

78. Tessitore A, Santangelo G, De Micco R, Giordano A, Raimo S, Amboni M, et al. Resting-state brain networks in patients with Parkinson's disease and impulse control disorders. Cortex (2017) 94:63-72. doi:10.1016/j.cortex. 2017.06.008

79. Tessitore A, De Micco R, Giordano A, di Nardo F, Caiazzo G, Siciliano M, et al. Intrinsic brain connectivity predicts impulse control disorders in patients with Parkinson's disease. Mov Disord (2017) 32(12):1710-9. doi:10.1002/ mds. 27139

80. Evans AH, Pavese N, Lawrence AD, Tai YF, Appel S, Doder M, et al. Compulsive drug use linked to sensitized ventral striatal dopamine transmission. Ann Neurol (2006) 59(5):852-8. doi:10.1002/ana.20822

81. Steeves TD, Miyasaki J, Zurowski M, Lang AE, Pellecchia G, Van Eimeren T, et al. Increased striatal dopamine release in parkinsonian patients with pathological gambling: a [11C] raclopride PET study. Brain (2009) 132 (Pt 5):1376-85. doi:10.1093/brain/awp054

82. O'Sullivan SS, Wu K, Politis M, Lawrence AD, Evans AH, Bose SK, et al. Cue-induced striatal dopamine release in Parkinson's disease-associated impulsive-compulsive behaviours. Brain (2011) 134(Pt 4):969-78. doi:10.1093/ brain/awr003

83. Politis M, Loane C, Wu K, O'Sullivan SS, Woodhead Z, Kiferle L, et al. Neural response to visual sexual cues in dopamine treatment-linked hypersexuality in Parkinson's disease. Brain (2013) 136(Pt 2):400-11. doi:10.1093/ brain/aws326

84. Voon V, Gao J, Brezing C, Symmonds M, Ekanayake V, Fernandez H, et al. Dopamine agonists and risk: impulse control disorders in Parkinson's disease. Brain (2011) 134(Pt 5):1438-46. doi:10.1093/brain/awr080

85. Cilia R, Siri C, Marotta G, Isaias IU, De Gaspari D, Canesi M, et al. Functional abnormalities underlying pathological gambling in Parkinson disease. Arch Neurol (2008) 65(12):1604-11. doi:10.1001/archneur.65.12. 1604

86. Petersen K, Van Wouwe N, Stark A, Lin YC, Kang H, Trujillo-Diaz P, et al. Ventral striatal network connectivity reflects reward learning and behavior in patients with Parkinson's disease. Hum Brain Mapp (2018) 39(1):509-21. doi:10.1002/hbm.23860

87. Pellicano C, Niccolini F, Wu K, O'Sullivan SS, Lawrence AD, Lees AJ, et al. Morphometric changes in the reward system of Parkinson's disease patients with impulse control disorders. J Neurol (2015) 262(12):2653-61. doi:10.1007/s00415-015-7892-3

88. Biundo R, Formento-Dojot P, Facchini S, Vallelunga A, Ghezzo L, Foscolo L, et al. Brain volume changes in Parkinson's disease and their relationship with cognitive and behavioural abnormalities. J Neurol Sci (2011) 310(1-2):64-9. doi:10.1016/j.jns.2011.08.001

89. Ricciardi L, Lambert C, De Micco R, Morgante F, Edwards M. Can we predict development of impulsive-compulsive behaviours in Parkinson's disease? J Neurol Neurosurg Psychiatry (2017). doi:10.1136/jnnp-2017-317007

90. Cerasa A, Salsone M, Nigro S, Chiriaco C, Donzuso G, Bosco D, et al. Cortical volume and folding abnormalities in Parkinson's disease patients with pathological gambling. Parkinsonism Relat Disord (2014) 20(11):1209-14. doi:10.1016/j.parkreldis.2014.09.001

91. Biundo R, Weis L, Facchini S, Formento-Dojot P, Vallelunga A, Pilleri M, et al. Patterns of cortical thickness associated with impulse control disorders in Parkinson's disease. Mov Disord (2015) 30(5):688-95. doi:10.1002/ mds. 26154

92. Yoo HB, Lee JY, Lee JS, Kang H, Kim YK, Song IC, et al. Whole-brain diffusion-tensor changes in parkinsonian patients with impulse control disorders. J Clin Neurol (2015) 11(1):42-7. doi:10.3988/jcn.2015.11.1.42

93. Imperiale F, Agosta F, Canu E, Markovic V, Inuggi A, Jecmenica-Lukic M, et al. Brain structural and functional signatures of impulsive-compulsive behaviours in Parkinson's disease. Mol Psychiatry (2018) 23(2):459-66. doi:10.1038/mp.2017.18

94. Happe S, Baier PC, Helmschmied K, Meller J, Tatsch K, Paulus W. Association of daytime sleepiness with nigrostriatal dopaminergic degeneration in early Parkinson's disease. J Neurol (2007) 254(8):1037-43. doi:10.1007/ s00415-006-0483-6

95. Hilker R, Razai N, Ghaemi M, Weisenbach S, Rudolf J, Szelies B, et al. $[18 \mathrm{~F}]$ fluorodopa uptake in the upper brainstem measured with positron emission tomography correlates with decreased REM sleep duration in early Parkinson's disease. Clin Neurol Neurosurg (2003) 105(4):262-9. doi:10.1016/S0303-8467(03)00058-1

96. Barber TR, Klein JC, Mackay CE, Hu MTM. Neuroimaging in pre-motor Parkinson's disease. Neuroimage Clin (2017) 15:215-27. doi:10.1016/j. nicl.2017.04.011

97. Heller J, Brcina N, Dogan I, Holtbernd F, Romanzetti S, Schulz JB, et al. Brain imaging findings in idiopathic REM sleep behavior disorder (RBD) a systematic review on potential biomarkers for neurodegeneration. Sleep Med Rev (2017) 34:23-33. doi:10.1016/j.smrv.2016.06.006

98. Chung SJ, Choi YH, Kwon H, Park YH, Yun HJ, Yoo HS, et al. Sleep disturbance may alter white matter and resting state functional connectivities in Parkinson's disease. Sleep (2017) 40(3):zsx009. doi:10.1093/sleep/zsx009

99. Rahmani F, Ansari M, Pooyan A, Mirbagheri MM, Aarabi MH. Differences in white matter microstructure between Parkinson's disease patients with and without REM sleep behavior disorder. Conf Proc IEEE Eng Med Biol Soc (2016) 2016:1124-6. doi:10.1109/EMBC.2016.7590901

100. Ansari M, Rahmani F, Dolatshahi M, Pooyan A, Aarabi MH. Brain pathway differences between Parkinson's disease patients with and without REM sleep behavior disorder. Sleep Breath (2017) 21(1):155-61. doi:10.1007/ s11325-016-1435-8

101. Defazio G, Tinazzi M, Berardelli A. How pain arises in Parkinson's disease? Eur J Neurol (2013) 20(12):1517-23. doi:10.1111/ene.12260

102. Wasner G, Deuschl G. Pains in Parkinson disease - many syndromes under one umbrella. Nat Rev Neurol (2012) 8(5):284-94. doi:10.1038/ nrneurol.2012.54

103. Tracey I, Mantyh PW. The cerebral signature for pain perception and its modulation. Neuron (2007) 55(3):377-91. doi:10.1016/j.neuron.2007. 07.012

104. Garcia-Larrea L, Peyron R. Pain matrices and neuropathic pain matrices: a review. Pain (2013) 154(Suppl 1):S29-43. doi:10.1016/j.pain.2013.09.001

105. Apkarian AV, Bushnell MC, Treede RD, Zubieta JK. Human brain mechanisms of pain perception and regulation in health and disease. Eur J Pain (2005) 9(4):463-84. doi:10.1016/j.ejpain.2004.11.001

106. De Felice M, Ossipov MH. Cortical and subcortical modulation of pain. Pain Manag (2016) 6(2):111-20. doi:10.2217/pmt.15.63

107. Bingel U, Quante M, Knab R, Bromm B, Weiller C, Büchel C. Subcortical structures involved in pain processing: evidence from single-trial fMRI. Pain (2002) 99(1-2):313-21. doi:10.1016/S0304-3959(02)00157-4

108. Apkarian AV, Baliki MN, Geha PY. Towards a theory of chronic pain. Prog Neurobiol (2009) 87(2):81-97. doi:10.1016/j.pneurobio.2008.09.018

109. Brefel-Courbon C, Payoux P, Thalamas C, Ory F, Quelven I, Chollet F, et al. Effect of levodopa on pain threshold in Parkinson's disease: a clinical and positron emission tomography study. Mov Disord (2005) 20(12):1557-63. doi:10.1002/mds.20629

110. Brefel-Courbon C, Ory-Magne F, Thalamas C, Payoux P, Rascol O. Nociceptive brain activation in patients with neuropathic pain related to Parkinson's disease. Parkinsonism Relat Disord (2013) 19(5):548-52. doi:10.1016/j.parkreldis.2013.02.003

111. Tessitore A, Russo A, De Micco R, Fratello M, Caiazzo G, Giordano A, et al. Central pain processing in "drug-naïve" pain-free patients with Parkinson's disease. Hum Brain Mapp (2018) 39(2):932-40. doi:10.1002/hbm.23892

Conflict of Interest Statement: The author declares that the research was conducted in the absence of any commercial or financial relationships that could be construed as a potential conflict of interest.

The reviewer MG and handling Editor declared their shared affiliation.

Copyright (C) 2018 Prell. This is an open-access article distributed under the terms of the Creative Commons Attribution License (CC BY). The use, distribution or reproduction in other forums is permitted, provided the original author(s) and the copyright owner are credited and that the original publication in this journal is cited, in accordance with accepted academic practice. No use, distribution or reproduction is permitted which does not comply with these terms. 